OVARIOTOMY : REMOVAL OF AN ENCYSTED TUMOUR OF THE LEFT UTERINE APPENDACBES.

By George Southax, Surgeon to the Salford Royal Hospital and Dispensary, Manchester.

(Read at the Anniversary Meeting of the Provincial Medical and Surgical Association, at Sheffield, Thursday, July 31, 1845.)

Early in June, Mrs. S., aged 38, called upon me and stated that she was suffering from an ovarian tumour, which having resisted $a$ variety of renedial means, she was extremely decirous of having it extirpated. The following is a brief sketch of her history :-

She has been married twenty years, never had any family, and until within the last few years has generally enjoyed good health. She noticed an increase in the size of her abdomen eight years ago, but as it was not attended with any derangement in her health, or irre gularity in the menstrual function, it was attributed to corpulency. Some time having elapsed, and there being evident deterioration in her condition, medical aid was requested. Since then she has been under the care of several experienced practitioners, and numerons remedies have been resorted to, all of which, with the exception perhaps of retarding the progress of the disease, have proved ineffectual. During the last twelve montbs the tumour has acquired such a size as to interfere with respiration, especially when in the recumbent posture, and she has occasionally suffered from pains in the left inguinal region. Tapping has been frequently recomn,ended to relieve these symptoms, but she has bitherto refused to accede to it. No apparent reason can be given for the disease, unless it originated from a blow in the abdomen when about 16 years of age; nor is she aware that the tumour began in either side, not having noticed it until a uniform swelling above the pubes presented itself.

On examining the abdomen I found it nearly globular, and very prominent, distended to at least twice the size of a female at the full term of pregnancy, and elevating the cartilages 'of the ribs to a considerable extent; the parietes were perfectly smooth and natural in colour; fuctuation, though of a resisting kind, was very distinct in every part, and percussion elicited a dull sound. Change of posifín produced scarcely any alteration in its form. The uterus, as examined per vaginam et rectum, seemed to be of the natural size, but was situated higher in the pelvis than usual; the os was quite healthy, and on the abdomen being raised and depressed by an assistant, whilst the finger was placed in immediate contact with it, the uierus uright be distinctly felt to rise and fall with an inclination to the left side. In the erect position it could not be made to bound away from the finger. There was no protrusion of the vaginal parietes, but an elastic swelling could be easily perceired, pressing on the left side of the uterus and upper part of the vagina; in other respects the pelvic cavity was not encroached upon. Though somewhat emaciated, her general health did not appear impaired; appetite good; tongue clean; pulse natural; bowels occasionally constipated, but easily acted on by medicine; catamenia regular, though in less quantity than formerly; felt pain at times in the left inguinal region, or whenerer she lay on the left side, and in consequence of the pressure of the tumour upwards, she has been unable to lie on her back, or in any other position, excepting on the right side, for the last twelve montlss.

The symptoms clearly indicating encysted dropsy of the left uterine appendages, adranced to such * stage as to render surgical interference necessary, and her constitution not being much affected, I considered the case was peculiarly farourable for the operation. At the same time, in giving this opinion, I candidly stated that tapping, which was now absolutely required, though merely palliative, was attended with less risk, and would probably prolong her life in tolerable comfort for some years. Extirpation was, howerer, the only effectual means of radically remoring the disease, but much more dangerous, two out of five of those who liad submitted to it haring died. Being possessed of good moral courage, and having witnessed the complete restoration to health of others who had submitted to the operation, she stated her willingness to undergo it, notwitustarding its concomitant dangers. Considering it would be more satisfactory to her friends to have another opinion, I requested Dr. Radford to accompany me in my next visit, who, after a carefully conducted examination, coincided with $\mathrm{my}$ views.

Extirpation being determined upon, it was fixed to take place on Tuesday, the 24 th of June, and her residence not being in my immediate vicinity, I adrised her to take lodgings near me. The prelininary arrangements having been entered into, such as abstinence from animal food and stimulating liquors, for a few days, the free evacuation of the bowels with castor oil, the temperature of the room raised to $75^{\circ}$ Fahrenheit, and the bladder previously emptied, I commenced the operation at four o'clock in the afternoon, in the presence of Drs. Radford, and Clay, Dr. Watson, of Liverpool, Messrs. Robertson, Nursaw, Horne, and Winterbottom, surgeons, and Mr. Willianson, my 
pupil, all of whom promptly rendered whatever assistance was necessary. An exploratory incision, midway between the umbilicus and pubes, was first made, and the peritoneal cavity opened sufficiently to admit the finger. A characteristic membrane of a bluish white and shining surface appearing at the opening at once satisfied me of the existence of a cyst, and the finger introduced between it and the peritoneum, discovering no adhesions in the immediate neighbourhood, I punctured it with a full-sized trocar.

After from sixteen to eighteen pints of clear, lemoncoloured, slightly mucilaginous fluid had been evacuated, pressure on the parietes being well sustained during its escape, the canula was withdrawn, and using the index finger as a director, the opening was enlarged above and below with a probe-pointed bistoury to the extent of between six and seven inches. Having ascertained by the hand introduced into the abdominal cavity, that there were no impediments to the extraction of the tumour, it was carefully drawn out, gentle pressure being continued on the abdomen. Finding it was attached to the uterine extremity of the left broad ligament by a short and slightly vascular pedicle, I tied it firmly with a single ligature of the strongest dentist's silk. 'The pedicle -was now divided, and the tumour being removed, the margins of the wound were immediately approximated to prevent the ingress of air. After a brief interval, the wound was again opened to remove what blood had escaped internally, and to ascertain that the vessels of the broad ligament were firmly secured. The uterus and opposite ovary were also examined and found healthy. One end of the ligature being cut off, the other was left dependent at the lowest point of the wound, the eilges of which were brought together by four interrupted sutures and straps of transparent tissue plaster. Upon these a broad pad was applied, and the whole being adjusted by a bandage, the patient was lifted into bed, within twenty-five minutes from the commencement of the operation. During the whole time she sustained herself remarkably well, apparently not suffering much pain, except whilst the exploratory incision was made, during which some little delay occurred, from the parietes being freely supplied with adipose tissue. No difficulty was experienced from the intestines protruding, as they were remarkably flaccid. There was very little hæmorrhage and no vomiting, but a feeling of faintness was superinduced by the evacuation of the fluid.

On being placed in bed she complained of pain in the left iliac region and back; pulse firm, 88. I ordered a grain of the acetate of morphia in the form of pill; toast-water in small quantities when thirsty ; and the temperature to be kept at $70^{\circ}$ Fahrenheit.

Half-past seven P.M. Pulse soft, 108; slept three quarters of an hour after the pill; pain in the side continues; with the exception of a little retching after taking the pill, there has not been the slightest disposition to romit. Says she is both hungry and thirsty. To be restricted to toast-water.

25tb. 3 A.M. Has bad come pain along the cQurse of the wound and in the left side during the last hour; pressure on the abdomen increases it; complains of a feeling of distention in the bowels, with frequent desire to pass flatus, which she is unable to do. Skin bot and dry; no vomiting; tongue assuming a mor- bid redness; has not slept since the last visit. As the pulse was fuller and more frequent, I took fourteen ounces of blood from the arm, which had the effect of lowering it twelve pulsations, and causing faintness. The night being warm the temperature of the room to be reduced to $67^{\circ}$, and two thirds of a grain of morphia administered.

Half-past 8 A.M. Parted with flatus frequently during the last three hours, which has relieved the pain in the abdomen. Pulse 116, soft ; skin moist, and of the ordinary temperature; passed about twelve ounces of urine since last visit; still thirsty and feels hungry; appears to be proceeding very favourably.

11 A.M. Visited with Dr. Radford, and found her in the same comfortable state as when last seen; pulse 108 ; temperature $68^{\circ}$. To have barley water, with half an ounce of gum dissolved in each pint.

5 P.M. Vomited once since last visit, which she attributed to some tea she had taken; tongue moist, yet redder than natural; countenance cheerful, but features slightly contracted; pulse 116 ; passed eight ounces of urine; thirst continues. To be allowed a wine-glassful of soda water occasionally, and a biscuit.

11 P.M. No return of sickness; pulse 106, soft ; troubled with flatulence; expresses herself as feeling very comfortable; slept almost the whole of the time since last visit. The bandage being rather tight it was slackened.

26th, 7 A.M. Had a good night, having slept several hours; features still rather contracted, but cheerful pulse soft, 100 ; tongue red, rather dry, and furred down the sides; skin moist ; thirsty ; passed a pint of urine during the night; has taken half a biscuit this morning. Bowels not having been moved since the operation, to take half an ounce of castor oil.

11 A.M. Visited with Dr. Radford; the oil having produced no alvine evacuation to have a common enema. The bandage and pads were removed, and the wound as seen through the transparent plaster appeared to be healing.

10 P.M. The enema produced a copious evacuation, but previously had severe pain in the abdomen, which has returned at intervals during the afternoon. It appears to be caused by flatus. Features still contracted, but countenance cheerful ; pulse 104, soft ; tongue moister, still red and slightly furred; passed urine twice since last visit, which was rather high coloured and turbid; less thirst. To take ten grains of carbonate of soda in aniseed water every two hours, whilst the flatulence continues.

27th. 7 A.M. Passed a comfortable night, the draught having entirely relieved the flatulence; complains of a short cough, attended with expectoration; tongue moist and less red; pulse 100, soft; bowels moved about an hour ago; passed about eight ounces of high coloured urine through the night.

11 A.M. Visited with Dr. Radford; symptoms the same; the cough and expectoration continuing, ordered a mustard poultice to the chest; the day being cold and wet, to increase the temperature of the room to $70^{\circ}$.

Pulv. Ipecac. Co. gr. iij. tert. hor. capiend.

Diet to consist of barley water, biscuit, and tea.

3. P.M. No change in the synptoms; examined the wound, which looks remarkably healthy; cough continuing, only one ligature was removed. To repeat 
the mustard poultice; and as she is tired of barley water, to have sago.

11 P.M. Cough quite relieved since the last poultice, and she is now very comfortable; skin moist and warm; countenance cheerful; pulse 106; enjoyed her sago. To omit the pills.

June 28th. 8 A.M. Passed an excellent night, and feels comparatively well; tongue moist and natural in colour; pulse soft, 96 ; no cough; skin of natural temperature and moist; no thirst ; passed about twelve ounces of urine during the night, which is clear, but rather high coloured.

11 A.M. Visited with Dr. Radford. The same. To be allowed some veal broth.

11 P.M. Had a very comfortable day; feels well enough to sit up; features no longer contracted; pulse 94, soft ; passed urine freely since last visit, but had no evacuation.

R. Ol. Ricini. ozss. mane. cap.

29th. 10 A.M. Visited with Dr. Radford. Improving. The oil has acted once upon the bowels; pulse 92 ; tongue quite healthy in appearance. The wound being quite healed, except at the lowest point, the three remaining ligatures were removed.

8 P.M. The cough returned during the afternoon, but was immediately relieved by a mustard poultice and two pills, (pulv. ipecac. co.); pulse 90, soft; bowels moved this afternoon; no flatulence.

30th. 11 A,M. Visited with Dr. Radford, and found her progressing very favourably; passed an excellent night; feels hungry, and thinks she ought be allowed to sit up to day. Bowels moved late last night.

July lst. Passed a very comfortable night ; pulse 88 ; was out of bed this morning for the first time; felt very mazy, and had some difficulty in balancing herself. To be allowed some chicken broth for dinner, and bread ad libitum.

2nd. The cough returned last night, but was soon relieved by a poultice and the pills; did not sleep very well, owing to a pain in the epigastrium, which she attributed to having eaten too freely of the fowl; pulse 90 ; other symptoms those of health. Diet to consist of broth, fowl, sago, and tea.

3rd. Continues to improve; pulse 84; bowels moved daily without medicine; appetite good; appears to be suffering only from debility. 'To have a mutton chop for dinner, and to sit up for a short time.

5th. Gains strength daily; feels the want of some support to her chest when sitting up, although she is very tightly bandaged; is continually wanting food.

9th. Doing well; can walk across the room; catamenia appeared yesterday, but in small quantity.

12th. Returned home a distance of three miles, and bore the journey very well.

I have visited her at intervals up to the present period, and found her, on each occasion, much improved. The ligature* came away on the forty-ninth day, after which the fistulous opening closed. The cicatrix of the

- On referring to the report of my former patient, (Mfedical Gaxette, November, 1813,) it will be found that the tympanitis and obstinate vomiting which supervened on the operation, caused the ends of the ligatures to be drawn within the abdominal cavity ; the wound had perfectly healed, and the patient was restored to health, whilst they were still in the abdomen. After several weeks had elapsed, a small abscess appeared at the lower part of the cicatrix, an opening into which gave exit to a quantity of healthy pus entire wound does not measure more thanfour inches. She has gained flesh, and is now in the efjoyment of perfect health.

\section{DESCRIPTION OF THE DISEASE.}

The tumour consisted of one entire cyst, which, when distended with fluid, resembled the shape of an egg, and weighed thirty-one pounds; the larger end occupied the lower portion of the abdomen, and was highly rascular; the smaller one filled the epigastrium, presented no trace of vessels, and was so exceedingly diaphanous, that the colour of the fluid might be clearly distinguished through its parietes. The disease had evidently originated in the fold of the broad liga ment, the ovary being merely adherent to it. The structure of the cyst varied in different parts, being half an inch in thickness near its connexion with the broad ligament, and gradually becoming thinner towards the upper portion, where it appeared scarcely capable of sustaining the weight of the contained fluid, and in all probability would have been ruptured with a very slight blow. The interior presented all the characters of a secreting surface, and the whole of the larger extremity was studded with numerous little nodules, about the size of millet seeds, some in clusters, others solitary. Several appeared to have the consistence of cartilage, others were composed of calcareous matter. A small cyst was also found in the orary, which, in other respects, presented no abnormal characters.

It would be infringing on the objects of this meeting were I to make any lengthened remarks on the numerous disputed points which ovariotomy involves; I shall not therefore trespass on your time with any other observations than what the case calls for.

Difficulty in the diagnosis of ovarian tumours is perhaps the most insportant objection that can be urged agrinst the operation. This will no doubt continue so long as the mere history of the disease and an abdominal exanination are regarded as sufficient to detect its existence. In the case before us, although it had been of many years duration, and several experienced practitioners had been consulted, no raginal. examination had been made or proposed. In its earlier stage a circumscribed tumour might probably have been easily distinguished through the abdominal parietes, but when the patient applied to me the enlargement was so uniforu and symmetrical, accompanied with such distinct fluctuation in every part, that it was imposssible to say with any degree of certainty whether the fluid was encysted.

An examination per vayinam et rectum, cleared up all doubts, not only of the existence of an ovarian tumour, but of its exact nature. The absence of any protrusion of the vaginal parietes, the elevated position of the uterus in the pelvic cavity, and the inability to cause it to bound away from the finger, proved that the fluid could not be ascitic, whilst the projection of

and the ligatures. $\Lambda$ frec discharge was promoted by poultices for a fow days, and at the end of a week the wound closed. No constitutional disturbance occurred, and there has not been the slightest interruption to the most perfect state of health since the termination of the report. The catamenia appear with the greatest regularity, and in the same quantity as previously to the commencement of the disease. There is a tendency to corpulency, which is in a great measure checked by her active habits. 
the swelling on the left side, the decided influence which raising and depressing the abdomen produced on the position of the uterus, with its inclination to one side more than the other, evidently indicated that the disease was connected with the left uterine appendages. The uniformity in the abdominal distension, the distinct fluctuation in every part of it, and the inability to discover.any solid matter encroaching on the pelvic cavity, led me to infer that it consisted of one or two cysts only; and the comparative intmunity from any great degree of suffering until within the last twelve or eighteeen months, the absence in the history of any previous attacks of peritonitis, together with the slight impediment to the action of the bowels, rendered the existence of ahhesions doubtful.

In the operation, the evils of both the major and minor incisions were guarded against, by making the opening no larger than was necessary to ascertain the nature and connexions of the disease, and to admit of its remoral after reducing it by paracentesis, without occasioning the least violence or displacement to any of the neighbouring parts. Had the slightest obstacle occurred previous to the successful termination to the operation, this plan would also have enabled me to have receded without much danger to the patient. Every stage of it, however, fully confirmed the accuracy of the diagnosis, and the trifling interference required in the subsequent treatment contributes to make this one of the most successful cases yet recorded. Medicine having failed in clecking the disease, its interference with respiration, and embarassment to the patient in pursuing her ordinary occupations, pointed sut the necessity for surgical aid. The inadequacy of tapping, save for temporary relief, the fuvourable nature of the disease, and the heulthy condition of the patient, induced me to undertake its extirpation, which I should hardly have sanctioned at an earlier period; for in the present state of our experience, the mere existence of an ovarian tumour should not lead us to decide on its remoral, uutil the inconveniences have become so great as to render surgical interference indispensible. At the same time it would be highly improper to delay its performance until the patient's constitution has been inraded, or some important organ implicated. Where the diagnosis is not sufficiently evident, as a preliminary measure, recourse may be had to tapping, which will frequently clear up any uncertainties that may exist, or if the system has become enfeebled from the pressure and weight of the accumulated fluid, it will afford time for its renovation. From the tumour presenting that form of ovarian disease, for which tapping is the most beneficial, it will perhaps be urged, that the patient ought not to have undergone the risks of the more formidable operation. Life is no doubt often prolonged by paracentesis. But how frequently is the brief interval of relief thus afforded, counterbalanced by the increased distress attending its repetition, which, from producing a continual drain on the system, sooner or later shatters the constitution, and after protracting the individual's sufferings, terminates in death.

The first object of all surgical operations is of course the preservation of life. If this can be gained more fectually by tapping, notwithstanding its disadranages, it ought to be preferred to extirpation. Looking merely at the average mortality of the cases in which the latter has been undertaken, the risk certainly appears very great. Since, however, in addition to every variety of disease, from the simple encysted dropsy, to the most malignant form of carcinoma, it comprises all those in which the operation has been raslily and unnecessarily performed, it cannot be considered applicable to particular cases. Indeed, a perusal of them plainly shows that the encysted form is not only the most favourable for extirpation, but where the cases have been selected in accordance with the rules which direct us as to the propriety of other operations, and no unnecessary violence has been used in removing the tumours, the mortality has been less than that of major operations generally.

The indifference evinced by many to investigate the researches of others, no doubt forms a strong incentive to test the value of a remedy by statistics; but when this mechanical system of reflecting is allowed to usurp the power of reason or of knowledge, acquired by experience and observation, or "confines itself to correcting facts without interpreting them,"* the principles founded on it will be liable to error.

The unqualified opposition to ovariotomy, formed chiefly from statistical data, by some eminent individuals, appears singularly inconsistent, since they do not hesitate to undertake operations still more dangerous for the removal of diseases which are also not immediately destructive to life; I allude to the application of a ligature to the innominat 4 , and other large arteries, for aneurism, the former of which, though performed several times by the ablest surgeons, has, I believe, invariably failed.

When medical education was less general, the weight of a great name might have influenced the estimation of any new doctrine by the profession, but the mind of erery practitioner is now so well stored with information, that the opinions of no man, however distinguished, are likely to be received with any farour, unless founded on a proper investigation of the facts which the subject en.braces.

Orariotomy, combining as it does so many requisites for its successful prosecution, is not calculated to find general favour in the eyes of so divided a profession as the medical.

To the skilful treatment of the physician, must be superadded the tact and promptitude in emergencies of the operative surgeon, as well as the experience of the accoucheur in uterine and abdominal diseases. In proportion as the operator is deficient in any of these general attainments, he is liable to incur the blame of rashness or inhumanity, where, under more farourable auspices, he might have earned high encomiums for his efforts in arresting protracted suffering and disease. The apparent facility of the operation may induce persons to undertake it who are not qualified to form a correct diagnosis of the cases in which it is most likely to be beneficial. Mere manual dexterity, unaccompanied by a careful consideration of the circumstances of the case, and the condition of the patient, never can lead to the establishment of ovariotomy on a firm foundation. On the contrary, the rash and indiscriminate use of the knife may have a tendency to throw discredit on what might otherwise prove a valuable resource for eradicating a disease, which, to the most interesting portion of the human

- M. Combe. De. la Medicine en France, et en Italia Paris, 184?. 
race, has hitherto formed a serions obstacle to health and the enjoyment of life. Where the success of the operation is followed by such satisfactory results, some degree of risk may justifiably be incurred on the principle and advice of the father of medicine.

"Ad extremos morbos extrema remedia."

4, Crescent, Salford, August, 1845.

\section{RESEARCHES ON THE COMPOSITION OF THE BLOOD IN HEALTH AND IN DISEASE : A MEMOIR PRESENTED TO THE ACADEMIE DES SCIENCES, PARIS, Nor. 18, 1844.}

By A. Becquerel, M.D., and. A. Rodier, M.D.

(Translated from the Gazette Médicale de Paris, by Edgar Sheppard, Esq., M.R.C.S.)

(Continued from page 470.)

ON THE COMPOSITION OF THE BLOOD IN PARTICULAR DISEASES.

We can only examine the composition of the blood in a certain number of the diseases which are most commonly met with in practice, since there are many in which blood-letting is never: indicated, and has neverbeen prescribed. We shall proceed therefore to demonstrate that by means of the general principles before established, all the special phenomena which present themselves may be explained.

$$
\text { OF THE BLOOD IN TYPHUS FEVER. }
$$

Typhus fever may be considered in some measure, as the type of the extensive class of pyrexiæ. From the great variation which it presents in the nature and intensity of its symptoms, in its progress, and in its duration, it is well calculated for the study of the composition of the blood in these diserses, and of the modifications which the important influences of adynamia and ataxia may exercise on this fluid.

MM. Andral aud Gavarret have made numerous analyses on this subject, and have obtained results which may be summed up as follows:-In typhus fever the proportion of the globules is sometimes normal, 0.127 ; sometimes it becomes increased, and attains the degree which it ordinarily presents in plethora. This latter result they explain by admitting that typhus fever is very frequently developed in plethoric individuals.

The solid materials of the serum do not undergo any remarkable changes in their proportions.

The fibrin is never augmented, except there be a complication with some of the phlegmasiæ; it frequently remains normal, but frequently also it diminishes in a very extraordinary manner; this is particularly the case when the disease is severe, when it developes an adynamic state, and when there is a tendency to the production of hæmorrhages.

Let us now see what are the results at which we have arrived, and whether they differ from those obtained by these able observers.

We have analysed the blood of thirteen individuals suffering from typhus fever-eleven men and two women. Of the eleven men six were bled once, four twice, and one-three times. One of the women was bled once, the other three times, making altogether twenty-one blood-lettings.

The ages of the males were:-one 17 , two 18 , one
21 , one 22 , three 23 , one 28 , one 31 , and one 48 years. Their avocations, which it is unnecessary bere to mention, were various. None of them had dwelt in Paris from infancy, and the period of their arrival there, with one exception, was not very long previous to the attack of fever. One of them had lived in the metropolis for 8 , one for 11 , two for 12 , four for 18 months ; gne for 2 years, and one from 7 to 8 years. They were all of good strong constitution.

On entering into details we find that six were strongly made, and of a certain degree of em:onpoint; three were a little less strong, and two, although meagre in appearance, were strong also. All had previously enjoyed excellent health. One only, for the last year of his residence in Paris, had been subject to diarrhœa.

The following were the most prominent characters of the disease:-In three cases it was very severe, and two of the patients fell victims to it; in six cases it was of medium severity; in two it was slight. The fever assumed the following forms:-In the three severe cases, the disease presented itself in the adynamic form; in two out of the six of moderate intensity, the form was also adynamic; in the two slight cases it presented the so-called inflammatory type.

We shall examine separately the composition of the blood in the first, the second, and the third bloodlettings.

FIRST BLOOD-LETTINGS MADE IN ELEVEN MEN.

These first blood-lettings indicate the actual composition of the blood in typhus fever. They were made at the following periods of the disease :-Once on the seventh day, four times on the eighth, twice on the ninth, once on the tenth, twice on the twelfth, once on the fourteenth: The subjoined table represents the mean composition in these eleven blood-lettings.

MEAN COMPOSITION OF THE BLOOD IN ELEVRN CASES OF TYPHUS FEVER.

Deusity of the blood deprived of its ? fibrin ........ $\}$ - of serum . . . . . . 1025.4

Water . . . . . . . 797.

Globules . . . . . . . 127.4

Albumen . . . . . . . 64.8

Fibrin . . . . . . . . 2.8

Extractive matters and free salts $\quad 6.3$

Fatty matters. . . . . . . . 1.773

Seroline . . . . . . . Variable.

Phosphorated matter . . . . $0: 471$

Cholesterine . . . . . . . 0.089

Soap . . . . . . . . . 1.093

IN 1000 GRAMMES OF CALCINED BLOOD.

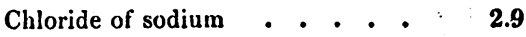

Soluble salts . . . . . . $\quad{ }^{2} 2.5$

Phosphates . . . . . . $\because 00.497$

Iron . . . . . • . • • • 0.555

The examination of this table, and of each case in particular, leads to the following conclusions :-

The globules, in typhus fever, are influenced in the same manner as we have seen them in every severe disease-that is, they experience a very marked diminution in quantity. There are, however, two circum- 